Rapid Reviews COVID-19

\title{
Review 1: "Assessment of Airborne Disease Transmission Risk and Energy Impact of HVAC Mitigation Strategies"
}

\section{Cary Faulkner ${ }^{1}$}

${ }^{1}$ University of Colorado Boulder

Published on: Jan 26, 2022

License: Creative Commons Attribution 4.0 International License(CC-BY 4.0). 


\section{$\underline{\text { RR:C19 Evidence Scale rating by reviewer: }}$}

- Reliable. The main study claims are generally justified by its methods and data. The results and conclusions are likely to be similar to the hypothetical ideal study. There are some minor caveats or limitations, but they would/do not change the major claims of the study. The study provides sufficient strength of evidence on its own that its main claims should be considered actionable, with some room for future revision.

$* * * * * * * * * * * * * * * * * * * * * * * * * * * * * * * * * * * * * * *$

\section{Review:}

Based on the Strength of Evidence Scale, the information in this manuscript is reliable. The authors compare different mitigation strategies for indoor virus based on equivalent outdoor air (EOA) and energy impact using an EnergyPlus model. Both EOA and energy impact are valuable metrics to provide guidance for building operators during the COVID-19 pandemic, since EOA gives an idea of the indoor air quality and energy impact determines how sustainable the solution is. The developed EnergyPlus model is sufficient to quantify these metrics for the different studied climates and the results provide some insightful information. The chosen mitigation strategies are common strategies of interest to improve indoor air quality during the COVID-19 pandemic. The results are clearly presented and the conclusions of the manuscript are backed up by the results.

The limitations of this study are reasonable. For example, assuming the zones are well mixed is a fair and common assumption in this field of work. Studying more scenarios, e.g. different building types, more levels of filtration/ventilation, etc. would be beneficial, but the current study provides insight with the cases performed. Metrics such as predicted number of infections would be interesting to see for this study, but EOA is sufficient based on the length of the paper. Lastly, EnergyPlus is a powerful tool for building simulation and energy analysis, but may ignore some of the fast system dynamics that could potentially affect the results.

Overall, the results of this study are not necessarily surprising and confirm the results of similar studies. This study provides some interesting insight and quantification for comparing these mitigation strategies, but more cases and analysis should be performed if this were to be submitted as a journal paper. 
